\title{
Possible assistance of host microbiome in SARS-CoV-2 fitness
}

Patrick SLAMA

Funding: The author(s) received no specific funding for this work.

Potential competing interests: The author(s) declared that no potential competing interests exist.

\begin{abstract}
The COVID-19 outburst that has immobilized a large part of the world originated in late 2019. It was associated with a bat-carried SARS-like virus, SARS-CoV-2. Many features of the disease nevertheless leave physicians and hospitals puzzled with respect to known viral infections. One issue is the discontinuous detection of viral RNA among patients, as well as its differential detection in faeces with respect to respiratory tract samples. Discontinuous detection will raise problems when cities and countries return to full activity, and already has in South Korea, where it was proposed that about one patient out of seven could become re-infected. A possible explanation for this proposed 're-infection' would be interactions of the virus with its host's microbiome.
\end{abstract}

A possible rationale for the discontinuous detection of the virus ${ }^{[1]}$ and its extended presence in faeces samples can be found exploring existing sequencing data ${ }^{[2]}$. In some RNA-seq analyses, bacteria from geni Prevotella and Veillonella are indeed present ${ }^{[3]}$ , often in much more abundant amounts than SARS-CoV-2 RNA. Chakraborty recently suggested this could provide some explanations regarding the 're-infection' phenomena and discontinuous RNA detections ${ }^{[4]}$.

Here we propose that an interaction between bacteria and the virus is indeed involved in the observed virulence, but not through the phage mechanism alluded to by Chakraborty, which seems unlikely owing to the known genetics of SARS-CoV-2. The hypothesis that those, or other gut commensal bacteria, could increase the infectivity of the SARS-CoV-2 virus, is indeed supported by existing knowledge on a possible facilitation of viral infection by bacteria. In mouse, it was shown that animals with antibiotics-depleted gut flora were less susceptible to poliovirus disease, with poliovirus binding bacteria through their lipopolysaccharide molecules ${ }^{[5]}$. Co-infection of coronavirus PEDV with Chlamyidiaceae was 
also observed in cell cultures, with larger inclusions in co-infected cells ${ }^{[6]}$. In humans, coinfection by Epstein-Barr virus in Mycoplasma pneumoniae patients was recently shown to increase symptoms duration, with increased levels of CRP and IL-2 ${ }^{[7]}$.

This view would also fit with other observations performed on COVID-19 patients and already described as symptoms for a gut bacterial infection, such as severe gut symptoms for multiple patients ${ }^{[8]}$, a burst in immune response, including an increase in Interleukins and cytokine ${ }^{[9]}$, as well as an observed decreased bioavailability of iron ${ }^{[10]}$ .The present hypothesis further enables one to understand the higher reliability or longer presence of SARS-CoV-2 RNA in faeces as opposed to oral or nasal swabs, with favorable interaction with gut bacteria[2]. The high rate of false negatives of patients dismissed from hospitals, with syndroms later re-emerging, could also be related to such an interaction ${ }^{[1]}$. In addition, an involvement of Prevotellae would match the higher prevalence of COVID-19 among overweight persons, this genus being over-represented in their gut microbiome ${ }^{[11]}$. Finally, this hypothesis would match the observation of symptoms both in the lungs and guts of patients. Prevotellae are indeed associated with increased inflammation in chronic obstructive pulmonary disease, asthma and cystic fibrosis, along with Veillonellae ${ }^{[12]}$, as well as in the guts of mice with provoked colitis ${ }^{[13]}$.

A rationale for a dysregulation of gut flora due to SARS-CoV-2 can be found in the literature by exploring the physiological roles of its main target, ACE2. In the intestinal epithelium, ACE2 assists in the uptake of amino-acids by an Slc6-family transporter ${ }^{[14]}$ , and in the biosynthesis of antimicrobial peptides ${ }^{[15]}$. Inhibition of ACE2 by SARS-CoV-2 would thus lead to an alteration of gut flora and diarrhea, a frequent COVID-19 symptom ${ }^{[16]}$, and to anemia, a predominant pathology among some COVID-19 cohorts $^{[17]}$.

Overall, the combination of an inhibition of the widespread ACE2 protein with constructive interactions with gut and/or lung bacteria would cover many of the observed COVID-19related symptoms. This hypothesis must nevertheless await more extensive patients data to be or not validated. In the meantime, therapies with antibiotics, or with compounds that would help restore natural flora, such as probiotics or nicotinamide ${ }^{[15][18]}$, should be given more consideration, above all in patients with gastrointestinal symptoms.

\section{References}

1. a, b Yafang Li, Lin Yao, Jiawei Li, Lei Chen, Yiyan Song, Zhifang Cai. (2020). Stability issues 
of RT-PCR testing of SARS-COV-2 for hospitalized patients clinically diagnosed with COVID-19.J Med Virol. doi:10.1002/jmv.25786.

2. a, b Yongjian Wu, Cheng Guo, Lantian Tang, Zhongsi Hong, Jianhui Zhou, Xin Dong. (2020). Prolonged presence of SARS-CoV-2 viral RNA in faecal samples. The Lancet Gastroenterology \& Hepatology, vol. 5 (5), 434-435. doi:10.1016/s24681253(20)30083-2.

3. ^ Wu, Fet al. (2020). A new coronavirus associated with human respiratory disease in China.

4. ^ Chakraborty S. (2020). The 2019 Wuhan outbreak could be caused by the bacteria Prevotella, which is aided by the coronavirus - v.3.

5. ^ S. K. Kuss, G. T. Best, C. A. Etheredge, A. J. Pruijssers, J. M. Frierson, L. V. Hooper. (2011). Intestinal Microbiota Promote Enteric Virus Replication and Systemic Pathogenesis. Science, vol. 334 (6053), 249-252. doi:10.1126/science.1211057.

6. ' Stuedli A, Grest P, Schiller I, Pospischil A. (2005). Mixed infections in vitro with different Chlamydiaceae strains and a cell culture adapted porcine epidemic diarrhea virus. Veterinary Microbiol, vol. 106(3-4).

7. ^ Xu Y, LiS, LiuJ, ZhouJ, Jin F, Chen X, Wang Y, Jiang Y, Chen Z. (2020). Impact of Epstein-Barr virus coinfection in Mycoplasma pneumoniae pneumonia. Medicine (Baltimore), vol. 99.

8. ^ Lin L, Jiang X, Zhang Z, Huang S, Zhang Z, Fang Z, Gu Z, Gao L et al. (2020). Gastrointestinal symptoms of 95 cases with SARS-CoV-2 infection. Gut.

9. ^ Larsen, JM. The immune response to Prevotella bacteria in chronic inflammatory disease. Immunology, vol. 151(4) .

10. ^Skaar, EP. (2010). The battle for iron between bacterial pathogens and their vertebrate hosts. PLOS Pathogens, vol. 6(8).

11. 'Nirmalkar K, Murugesan S, Pizano-Zárate ML, Villalobos-Flores LE, García-González C, Morales-Hernández RM, Nuñez-Hernández JA, Hernández-Quiroz Fet al. (2018). Gut Microbiota and Endothelial Dysfunction Markers in Obese Mexican Children and Adolescents. Nutrients, vol. 10(12).

12. ^ Caverly LJ, Huang YJ, Sze MA. (2019). Past, Present, and Future Research on the Lung Microbiome in Inflammatory Airway Disease. Chest, vol. 156(2).

13. ^ Haange SB, Jehmlich N, Hoffmann M, Weber K, LehmannJ, von Bergen M, Slanina U. (2019). Disease Development Is Accompanied by Changes in Bacterial Protein Abundance and Functions in a Refined Model of Dextran Sulfate Sodium (DSS)-Induced Colitis.J Proteome Res, vol. 18(4).

14. ^ Lambert DW, Clarke NE, Turner AJ. (2009). Not just angiotensinases: new roles for the angiotensin-converting enzymes. Cell Mol. Life Sci., vol. 67. 
15. a, b Perlot T, Penninger JM. (2013). ACE2- From the renin-angiotensin system to gut microbiota and malnutrition. Microbes Infection, vol. 15.

16. `WeiXS, Wang X, Niu YR, Ye LL, Peng WB, Wang ZH, Yang WB, Yang BH et al. (2020). Diarrhea is associated with prolonged symptoms and viral carriage in COVID-19. Clin. Gastroenterol. Hepatol., vol. doi: 10.1016/j.cgh.2020.04.030.

17. `Zhang G, Hu C, Luo L, Fang F, Chen Y, LiJ, Peng Z, Pan H. (2020). Clinical features and short-term outcomes of 221 patients with COVID-19 in Wuhan, China. J Clin Virol, vol. 127:104364.

18. ^ Clayton PT, Bridges NA, Atherton DJ, Milla PJ, Malone M, Bender DA. (1991). Pellagra with colitis due to a defect in tryptophan metabolism. Eur. J. Pediatr., vol. 150(7) . 